\title{
Behavior of An Isolated Polymer Chain Residing in a Density Gradient of Segments
}

\author{
E. A. DiMarzio and C. M. Guttman \\ Institute for Materials Research, National Bureau of Standards, Washington, D.C. 20234 \\ (November 29, 1977)

\begin{abstract}
The $\mathbf{W}$ iener integral (path integral, functional integral) technique is used to determine the equation describing the probability distribution of a polymer molecule immersed in a non-uniform distribution of monomer units. This result should be useful whenever there is a spatial variation of polymer density such as at an interface or surface.

Key words: Functional integrals; path probability integrals; polymer conformations; polymer interface; polymer surface; Wiener integrals.
\end{abstract}

\section{Introduction}

Let us suppose that there is a region of space in which a spatially varying concentration of segments, $c(\mathbf{r})$ exists. We ask; what is the number of conformations and the shape of a polymer molecule confined to the same space (see fig. 1). Certain qualitative features are immediately apparent. The polymer segments will seek out the regions of low segment concentration $(c(\mathbf{r})$ small). In this way, the entropy will be maximized. Further, even if we pin the two ends of the polymer in high density regions the interior segments of the chain will tend to seek the lower density region. Thus, an effect exists and its results in distortions in polymer shape as well as a reduction of the number of conformations.

The effect exists whenever there is a spatial density variation of concentrated polymer. This is because any given polymer molecule can be viewed as immersed in a bath of the segments of the other polymer molecules that surround it. The solution of the problem of density variation in concentration polymer should proceed in two steps. The first step would be to solve the problem proposed here for an isolated polymer in a field of unconnected segments $c(\mathbf{r})$. Then, armed with this solution one would perform a (as yet unspecified) self-consistent calculation on the interacting molecules.

When polymers adsorb onto a surface from solution, they crowd each other and compete for space near the surface. Thus, the questions raised in the preceding paragraphs are of paramount importance for the surface adsorption problem. The treatment of isolated non-interfering polymers on a surface has been extensive and rather complete [1]. The treatment of interfering polymers on a surface is probably in its infancy, although several useful treatments on the problem exist [2]. Not many treatments exist which discuss shape changes at an interface $[3,4]$. Hoeve argues for exponential decay of individual chain density, but he has restricted himself to theta conditions [4]. For non-theta conditions the problem proposed here must first be solved.

\section{Solution to the Problem}

We shall solve the problem stated in the caption to figure 1 by relating it to a problem the solution of which is already known. This latter problem is that of a polymer immersed in a space with a potential $V(\mathbf{r})$ per segment so that for a given path specified by $\mathbf{r}(\tau)$ the total energy is given by

$$
E_{t}=\int_{0}^{t} V(\mathbf{r}(\tau)) d \tau
$$

where $t$ is the upper limit of $\tau$ which is a parameter labeling the segments of the macromolecule. The probability of a chain of length $t$ beginning at $\mathbf{r}_{0}$ and ending at $\mathbf{r}$, is

$$
\begin{aligned}
& P\left(\mathbf{r}, t ; \mathbf{r}_{0}, 0\right) \\
& \quad=\int \exp \left(-\frac{1}{k T} \int_{0}^{t_{0}} V(\mathbf{r}(\tau)) d \tau\right) d_{w\left(0, \mathbf{r}_{0} ; t, \mathbf{r}\right)} x
\end{aligned}
$$

where the operation $\int d_{w\left(0, \mathbf{r}_{0} ; t, \mathbf{r}\right)} x$ indicates an integration over all paths with $\mathbf{r}_{0}, 0$ and $\mathbf{r}_{0} t$ as end points. Equation (2) is nothing more than the Boltzmann weighting factor. Since it is also the Wiener integral, a connection between the statistical mechanics of a polymer molecule and a wealth of mathematical techniques is immediately made. The work of Brush contains a useful introduction to functional integral techniques [5]. Kac [6] has shown that eq (2) is the solution (Green's function, propagator) to the following equation

$$
\frac{\partial P}{\partial t}=D \nabla^{2} P-\frac{V(\mathbf{r})}{k T} P
$$

where at $t=0$ we have

$$
P\left(\mathbf{r}, 0, ; \mathbf{r}_{0} 0\right)=\delta\left(\mathbf{r}-\mathbf{r}_{0}\right)
$$




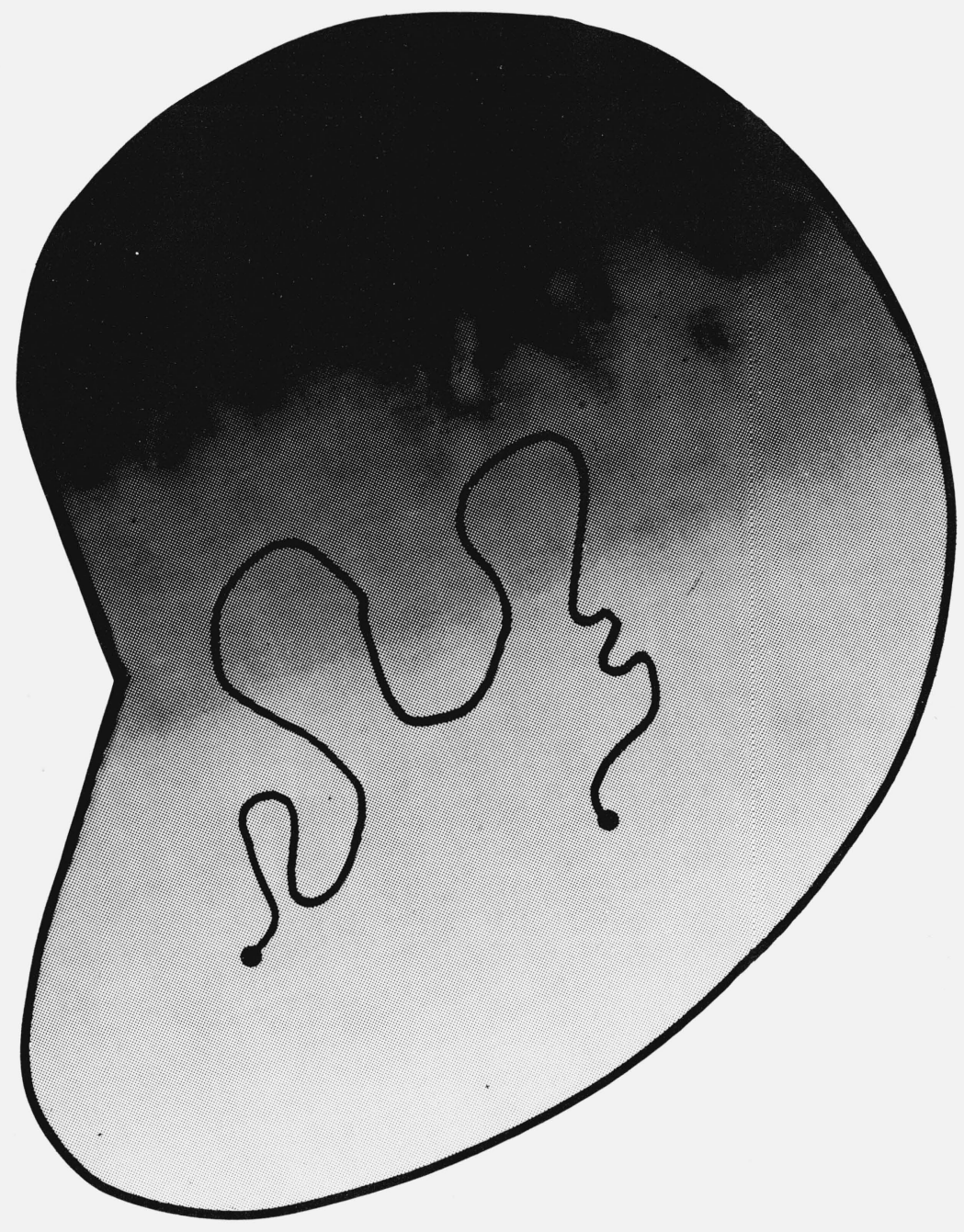

FIGURE 1. A polymer molecule of length $\mathrm{t}$ is placed into a region of space with a monomer concentration $\mathrm{c}(\mathbf{r})$ which is a function of position. The problem is to determine the effect of $c(\mathbf{r})$ on the probability distribution of the various allowed shapes of the polymer.

Equation 3 is recognized as the diffusion equation with annihilation except that $t$ represents the contour length of the chain as well as time and $D=\ell^{2} / 6$. Although the above relations were derived for the case of boundary conditions at infinity, for a boundary at finite places we have $[7,8]$

$$
P=0 \quad \text { on surface }
$$

This boundary condition has the effect of annihilating (and removing from further consideration) any chain that wanders across the surface. The conditions 3 completely define the problem and the solution $P\left(\mathbf{r}, t ; \mathbf{r}_{0}, 0\right)$ gives the probability of a chain of length $t$ whose end points are $\mathbf{r}_{0}$ and $\mathbf{r}$.

Let us now set up a correspondence between the volume fraction $c$ and $V$ for the case of no dependence on $\mathbf{r}$.

$$
V(\mathbf{r})=V_{c}, c(\mathbf{r})=c_{\boldsymbol{o}}
$$

It is easily verified by substitution that

$$
P=P_{0} \exp \left(-V_{c} t / k T\right)
$$

solves eq 3 when $P_{0}$ is the solution for $V(\mathbf{r})=0$ (the ordinary diffusion equation). Now $P_{0}$ is also a solution when $c=0$. The solution for the case of $c$, a constant is

$$
P=P_{0}\left(1-c_{0}\right)^{t}
$$

where we have used the ansatz that for each step the polymer has a success rate of $1-c_{0}$ (the volume fraction of emptyness). Note that the polymer is imagined to be infinitesimally thin since we are ignoring excluded volume. Equating (6) and (5) gives immediately

$$
1-c_{0}=\exp \left(-V_{c} / k T\right) .
$$

Thus, one solves the problem of a uniform distribution of segments by simply defining a $V_{c}$ by means of eq (7) and then using eqs (3) to find the probability distribution $P$. 
Having demonstrated the validity of the prescription for a constant distribution of segments we can easily demonstrate it is also valid when the distribution is non-uniform viz,

$$
1-c(\mathbf{r})=\exp (-V(\mathbf{r}) / k T) .
$$

To see this, observe that the weighting factor for a given path is

$$
\begin{aligned}
& \Pi_{\tau}(1-c(\mathbf{r}(\tau)) \\
& =\exp \left(+\int_{0}^{t} \ln (1-c(\mathbf{r}(\tau))) d \tau\right) .
\end{aligned}
$$

Comparing with the weighting factor of eq (2), we see that eq (8) immediately follows. Thus, the distribution of a polymer molecule in a field of a non-uniform distribution of segments is formally solved.

If one has both a potential $V_{e}(\mathbf{r})$ and a field of segments $c(\mathbf{r})$ then one solves the problem by using eqs (3) with a potential $V$ given by

$$
V(\mathbf{r})=V_{e}(\mathbf{r})-k T \ln (1-c(\mathbf{r})) .
$$

$V_{e}$ can, for example, represent a gravitational or centrifugal field.

De Gennes [9] and especially Edwards [10] have written extensively on the path integral approach to the solution of various polymer problems. The above result is contained implicitly in what they have done, but an explicit statement was never given. There are several persuasive reasons for an explicit display of eqs (3) and eq (8) as an exact prescription for solving a problem which may or may not be a subproblem in a larger effort (such as for example a self-consistent approach to the excluded volume problem.)

First, there is a strong intuitive appeal to the use of a term $-c(\mathbf{r}) P$ as an annihilation term in the diffusion equation. Several people have done it $[8,11]$. Because of its strong intuitive appeal, the realization of its approximate nature is sometimes forgotten.

Second, the approximation

$$
\ln (1-c(\mathbf{r}))=-c(\mathbf{r})
$$

has been used implicitly in self-consistent approaches to the derivation of a spacially dependent Flory-Huggins-like calculation [3, 12]. For high densities, this is not an adequate approximation.

Third, it enables us to attack the self-consistent field problem(s) as a two step problem thus simplifying matters. The effect of approximations are more readily assessed, especially since the part that we have given here is the exact part of the self-consistent approach. Also, there is pedagogical value in the above approach for those who are perhaps intimidated by the formalism of Edwards and De Gennes. Paths integral techniques are not usually included in the mathematical tool bag of most polymer scientists.

Finally, if the potential energy of eq (2) is time dependent as well as position dependent, eq (3) follows. This can be seen by examining the proof of Kac [6] and ascertaining that no step in the proof is invalidated by an assumed $t$ dependence. This means that eq (3) is immediately generalized to the case of time dependence of $c(\mathbf{r})$.

\section{References}

[1] For a list of References see the paper by DiMarzio, E. A., and Rubin, R. J., J. Chem. Phys. 55, 4318 (1971).

[2] Silberberg, A., J. Chem. Phys. 46, 1105 (1967); 48, 2835 (1968); J. Colloid Interface Sci. 38, 217 (1972); Roe, R. J., J. Chem. Phys. 60, 4192 (1974); 62, 490 (1975); Helfand, E., J. Chem. Phys. 63, 2192 (1975), Ash, S. G., Everett, D. H., and Findenegg, G. H., Trans. Faraday Soc. 64, 2645 (1968); 66, 708 (1970).

[3] Helfand, E., and Tagami, Y. Y., J. Chem. Phys. 56, 3592 (1972).

[4] Hoeve, C. A. J., J. Chem. Phys. 43, 3007 (1965); 44, 1505 (1966); J. Polym. Sci. 30, 361 (1970); 34, 1 (1971).

[5] Brush, S. G., Rev. Mod. Phys. 33, 79 (1961).

[6] Kac, M., Trans, Am. Math. Soc. 65, 1 (1949).

[7] Pouchly, J., Collection Czechoslov. Chem. Commun. 28, 1804 (1963).

[8] DiMarzio, E. A., J. Chem. Phys. 42, 2101 (1965).

[9] de Gennes, P. G., Rep. Prog. Phys. 32, 187 (1969).

[10] Edwards, S. F., Proc. Phys. Soc. 85, 613 (1965) Proc. Phys. Soc. 88, 265 (1966), Proc. Phys. Soc. 91, 513 (1967).

[11] James, H. M., J. Chem. Phys. 21, 1628 (1953).

[12] Dolan, A. K., and Edwards, S. F., Proc. Roy. Soc. London A343, 427 (1975). 